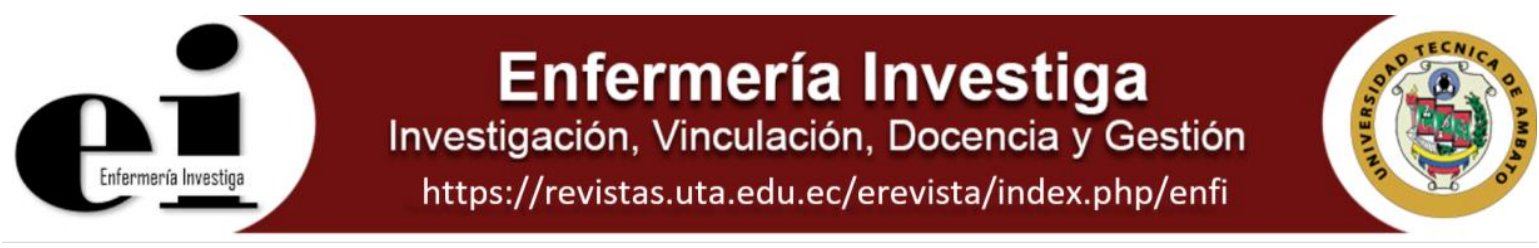

\title{
FACTORES ASOCIADOS A LA FALTA DE CONTROL PRENATAL EN AMÉRICA LATINA Y SU RELACIÓN CON LAS COMPLICACIONES OBSTÉTRICAS.
}

\section{FACTORS ASSOCIATED WITH THE LACK OF PRENATAL CONTROL IN LATIN AMERICA AND ITS RELATIONSHIP WITH OBSTETRIC COMPLICATIONS.}

\author{
Lourdes Jacqueline Barros Raza1 https://orcid.org/0000-0001-6317-4225, Evelin Fernanda Velasco Acurio ${ }^{2}$ \\ https://orcid.org/0000-0003-2732-6003.
}

${ }^{1}$ Estudiante de la Carrera de Enfermería, Universidad Técnica de Ambato, Ecuador.

2Docente de la Carrera de Enfermería, Universidad Técnica de Ambato, Ecuador

2477-9172 / 2550-6692 Derechos Reservados ( 2022 Universidad Técnica de Ambato, Carrera de Enfermería. Este es un artículo de acceso abierto distribuido bajo los términos de la Licencia Creative Commons, que permite uso ilimitado, distribución y reproducción en cualquier medio, siempre que la obra original es debidamente citada

Recibido: 01 de julio 2021

Aceptado: 20 de octubre 2021

\section{RESUMEN}

Introducción: El control prenatal es un conjunto de actividades y procedimientos que el equipo de salud ofrece a la embarazada, para la prevención de complicaciones, por medio del diagnóstico precoz de factores de riesgo en la gestante y enfermedades que puedan afectar el curso normal del embarazo y la salud del recién nacido. Objetivo: Identificar los factores asociados a la falta de control prenatal en América latina y su relación con las complicaciones obstétricas. Métodos: investigación de diseño documental con revisión bibliográfica en las bases de datos: PubMed, BVS, Scielo, Latindex, Lilacs y Google académico, con los descriptores de ciencias de la salud (DeCS) de Bireme y los Medical Subject Headings (MesH) de la National Library of Medicine: atención prenatal, embarazo, complicaciones del embarazo, salud materno-infantil, en idioma español e inglés durante el periodo 2015-2021. Resultados: La búsqueda inicial de información reportó un total de 1718 registros, de los cuales 86 eran elegibles, de estos 56 no fueron relevantes, por lo que quedan incluidos 30 que permitieron dar cumplimiento al objetivo de estudio con los cuales se procedió al meta-análisis. Conclusiones: Los factores asociados a la falta de control prenatal son: el bajo nivel de instrucción, ocupaciones del hogar y crianza, falta de empleo, escasos recursos económicos, ubicación geográfica, falta de afiliación, creencias y costumbres, estos factores se relacionan con complicaciones obstétricas como: infecciones, anemia, preeclampsia, hemorragias, desgarros perineales, alumbramiento incompleto, bajo peso y prematuridad.

\section{ABSTRACT}

Introduction: Prenatal control is a set of activities and procedures that the health team offers to the pregnant woman, for the prevention of complications, through the early diagnosis of risk factors in the pregnant woman and diseases that may affect the normal course of pregnancy. and the health of the newborn. Objective: To identify the factors associated with the lack of prenatal control in Latin America and its relationship with obstetric complications. Methods: documentary design research with bibliographic review in databases: PubMed, BVS, Scielo, Latindex, Lilacs and academic Google, with the descriptors of health sciences (DeCS) from Bireme and the Medical Subject Headings (MeSH) from the National Library of Medicine: prenatal care, pregnancy, pregnancy complications, maternal and child health, in Spanish and English during the period 2015-2021. Results: The initial search for information reported a total of 1718 records, of which 86 were eligible, of these 56 were not relevant, so 30 are included that allowed the fulfillment of the study objective with which the goal was carried out. analysis. Conclusions: The factors associated with the lack of prenatal control are: low level of education, home occupations and upbringing, lack of employment, scarce economic resources, geographic location, lack of affiliation, beliefs and customs, these factors are related to complications obstetrics such as: infections, anemia, preeclampsia, hemorrhages, perineal tears, incomplete delivery, low weight and prematurity.

Keywords: prenatal care, pregnancy, pregnancy complications, maternal and child health

Palabras claves: atención prenatal, embarazo, complicaciones del embarazo, salud materno-infantil 


\section{INTRODUCCIÓN}

Las complicaciones obstétricas debido al inadecuado control prenatal se consideran un problema de salud pública, por las cifras de morbimortalidad que ello desprende, pues de acuerdo a la Organización Mundial de la Salud, la mortalidad materna es inaceptable alta, cada día mueren en todo el mundo unas 830 mujeres por complicaciones relacionadas con el embarazo o parto, se estima que unas 303000 muertes de mujeres han muerto durante el embarazo, parto o puerperio, situación estrechamente relacionada con la atención sanitaria recibida durante el embarazo y el parto (1).

De acuerdo a reportes de la situación de morbilidad y mortalidad materna en América Latina y el Caribe, se han producido 7600 muertes maternas en el 2015 debido a hemorragias postparto e hipertensión, concentradas en los países con mayores tasas de fecundidad, pobreza y menor acceso a salud de calidad. Las estimaciones muestran que en Chile y Uruguay la mortalidad materna es menor 17 por cada 100.000 habitantes, también se reconoce que en países como Ecuador la mortalidad materna se ha reducido en un $50 \%$ pero en otros países como Venezuela se ha incrementado (2).

En el contexto latinoamericano, se reconoce con preocupación que miles de mujeres todavía pierden la vida cada año por causas prevenibles relacionadas con el embarazo y parto, muchas más sufren complicaciones y experimenta problemas de salud a largo plazo que afectan la calidad de sus vidas. Mujeres indígenas y afrodescendientes, así como mujeres aquellas con menores ingresos y menos años de educación formal a menudo carecen de acceso a los servicios de planificación familiar y asistencia prenatal, así también las mujeres gestantes indígenas de las Américas tienen tres veces más probabilidades de morir por causas relacionadas con el embarazo y el parto que las no indígenas debido a escases de servicios de salud (atención prenatal y perinatal) o creencias personales (3).

La dificultades de acceso a la atención prenatal, y la consiguiente pérdida de vidas, violan los derechos de la mujer a la salud debido a las múltiples complicaciones obstétricas que se presentan (4). De ahí que es importante analizar las causales del incumplimiento de controles prenatales con especial énfasis en Latinoamérica donde se reconoce que los recursos de salud son limitados y las condiciones sociales y económicas complejas, además que el incumplimiento de las consultas prenatales en una mujer gestante, implica múltiples riesgos que deben ser abordados por los gobiernos locales porque estos se desprenden de variables personales, familiares, sanitarios y sociales al ser elementos claves para la estructuración de los servicios de cuidado materno infantil (5).

Además, es necesario conocer, analizar y destacar todos los factores que influyen en la falta de control prenatal porque si bien en muchos casos esto se relaciona con condiciones individuales, sociales o económicas también guardan relación con aspectos de asistencia de salud y de tipo profesional entre los que se destacan: la oferta de servicios de salud, accesibilidad, costos y entre otros (6).

Por estas razones, se desarrolla el presente artículo con el objetivo de identificar los factores asociados a la falta de control prenatal en América latina y su relación con las complicaciones obstétricas para lo cual se analiza la importancia del control prenatal, las principales complicaciones obstétricas y finalmente se categorizan los factores sociodemográficos, actitudinales y sanitarios asociados a la falta de control prenatal, con lo que se desarrollará un documento sólido que permita ampliar el conocimiento en torno al tema para futuras investigaciones e intervenciones.

\section{MÉTODOS}

El tipo de estudio: revisión descriptiva (7), por medio de la estrategia PICO (por su sigla en inglés, Patiens-IntervetionCompare-Outcome-Study desing), se planteó la siguiente pregunta de investigación ¿La falta de control prenatal en las gestantes de América Latina se relaciona con el aparecimiento de complicaciones obstétricas? (8).

Protocolo de búsqueda y selección de artículos según las fases de la guía PRISMA (Preferred Reporting Items for Systematic reviews and Meta-Analyses) y con la participación de dos investigadores (9).

Identificación: en las bases de datos: PubMed, BVS Enfermería (Biblioteca Virtual en Salud Enfermería), Scielo, Latindex, LILACS y Google académico se realizó la búsqueda con descriptores en ciencias de la salud (DeCS) de Bireme y los Medical Subject Headings (MesH) de la National Library of Medicine en inglés, como: atención prenatal, embarazo, complicaciones del embarazo, salud materno-infantil, en idioma español e inglés.

Revisión: se realizó la extracción de la información de los artículos seleccionados por medio del análisis de títulos y resúmenes, verificando el cumplimiento de los siguientes criterios de inclusión: tipo de estudio: originales, revisiones sistemáticas, meta-análisis, relacionados con el control prenatal y las complicaciones obstétricas, realizados en América Latina, que abordaron las complicaciones obstétricas en periodo de embarazo, parto y puerperio tanto en la madre como en el neonato y en el periodo 2015-2021.

Elección: Los artículos elegibles fueron analizados en su texto completo para determinar con certeza que la información descrita es suficiente y apropiada para responder la pregunta de investigación, a fin de ser incorporada en la revisión sistemática y se les aplicaron los siguientes criterios de exclusión:

Inclusión: para la extracción de datos de cada uno de los estudios se utilizó una matriz con las siguientes características: autor, año, país, título y conclusiones.

Análisis de la información: Se emplearon dos modelos para combinar estadísticamente los resultados, el modelo de efectos fijos y el modelo de efectos aleatorios, lo cual permitió resumir de manera sintética los elementos.

\section{RESULTADOS}

En total se identificaron 1718 estudios que presentaron como tópico central el control prenatal y las complicaciones obstétricas. En la revisión se eliminaron 1632 investigaciones porque eran duplicados. Los 86 artículos restantes fueron revisados en texto completo y se excluyeron 46 , debido a que no fueron realizados en América latina, estuvieron fuera del idioma inglés-español, fueron publicadas fuera del periodo 2015-2020 y porque no fue posible acceder al texto completo. 40 estudios fueron sometidos a evaluación final de los cuales 10 se descartaron por no aportar al cumplimiento del objetivo y finalmente se realizó el meta-análisis con 30 artículos. (Fig. 1). 


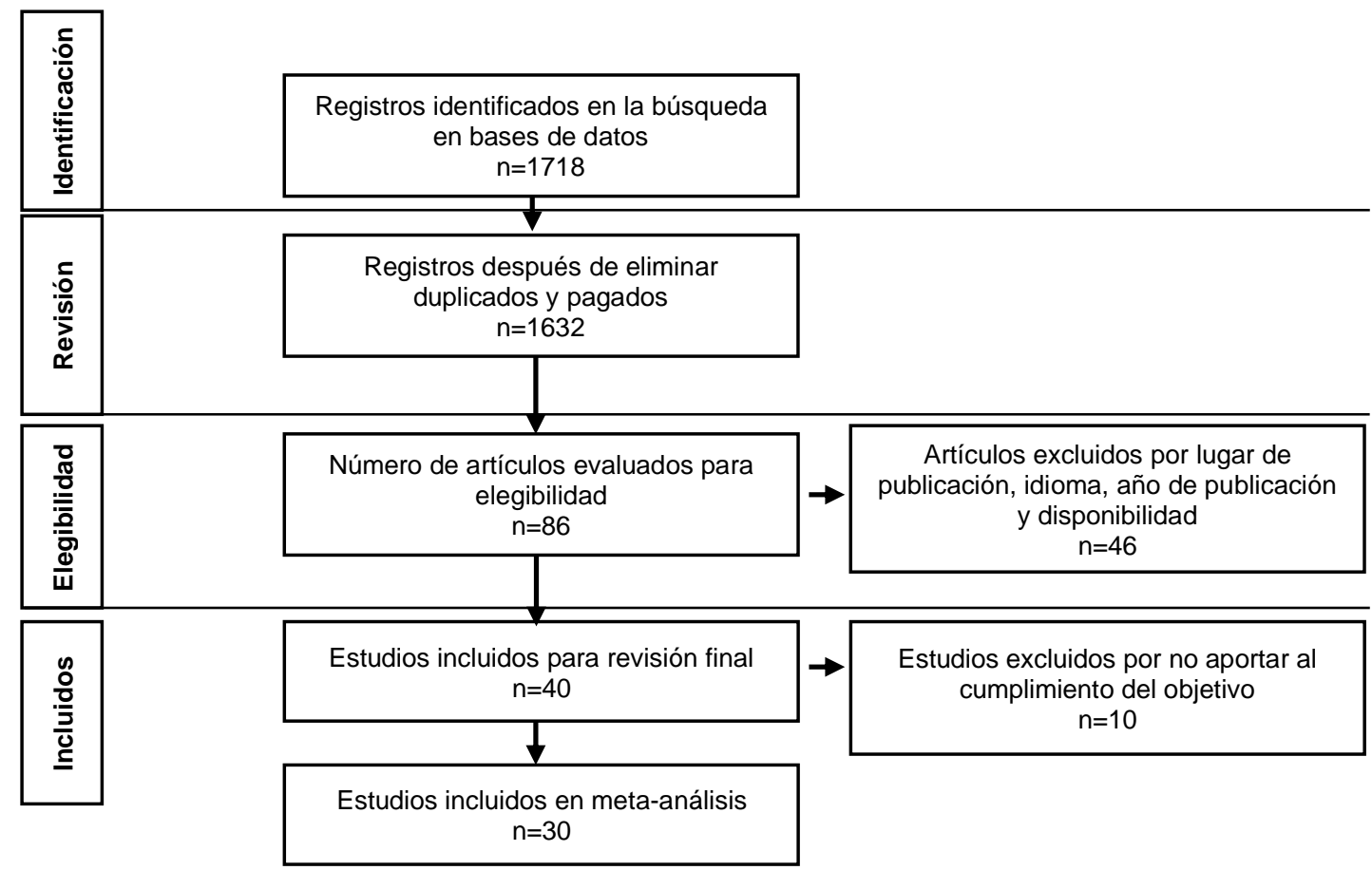

Tabla 1. Artículos científicos seleccionados para la revisión.

\begin{tabular}{|c|c|c|c|}
\hline Autor, año & País & Titulo & Conclusión \\
\hline $\begin{array}{l}\text { Núñez y col., } \\
2019\end{array}$ & Perú & $\begin{array}{l}\text { Complicaciones } \\
\text { obstétricas asociadas al } \\
\text { control prenatal } \\
\text { inadecuado en } \\
\text { puérperas atendidas en } \\
\text { un Centro materno } \\
\text { infantil peruano }\end{array}$ & $\begin{array}{l}\text { El } 39,39 \% \text { de las gestantes presentaron un } \\
\text { control prenatal incompleto, que originó: } \\
\text { inadecuada ganancia de peso, infecciones } \\
\text { urinarias y la anemia fueron las complicaciones } \\
\text { más prevalentes. El número de complicaciones } \\
\text { obstétricas es mayor en quienes tienen un control } \\
\text { prenatal inadecuado. }\end{array}$ \\
\hline $\begin{array}{l}\text { Cardozo y } \\
\text { col., } \\
2015\end{array}$ & Perú & $\begin{array}{l}\text { Complicaciones } \\
\text { obstétricas y morbilidad } \\
\text { obstétrica en gestantes } \\
\text { en el servicios Gíneco- } \\
\text { obstetricia en un } \\
\text { Hospital de } \\
\text { Lambayeque }\end{array}$ & $\begin{array}{l}\text { Las principales complicaciones obstétricas son: } \\
\text { hemorrágicas, infecciosas, hipertensivas y del } \\
\text { trabajo de parto, la morbilidad obstétrica fue de } \\
88,5 \% \text {, esto debido al incumplimiento de } \\
\text { controles prenatales mínimos recomendados. }\end{array}$ \\
\hline García, 2017 & Colombia & $\begin{array}{l}\text { Barreras de acceso y } \\
\text { calidad en el control } \\
\text { prenatal }\end{array}$ & $\begin{array}{l}\text { El } 74,17 \% \text { de gestantes tuvo cuatro o más } \\
\text { controles prenatales y pese a la alta cobertura } \\
\text { asistencial no se ha logrado el descenso de la } \\
\text { mortalidad materno perinatal. Las mujeres con } \\
\text { escaso o nulo control prenatal presentan } \\
\text { complicaciones que llevan a cesáreas de } \\
\text { emergencia }(25 \%) \text { o a la pérdida fetal }(15 \%) \text {. }\end{array}$ \\
\hline $\begin{array}{l}\text { Gómez y col., } \\
2017\end{array}$ & Colombia & $\begin{array}{l}\text { Impacto económico y } \\
\text { sanitario del control } \\
\text { prenatal en } \\
\text { embarazadas afiliadas } \\
\text { al régimen subsidiado } \\
\text { en Colombia }\end{array}$ & $\begin{array}{l}\text { Un buen control prenatal tiene un impacto } \\
\text { positivo sobre el desenlace del embarazo, la } \\
\text { mayoría de lesiones y muertes maternas, así } \\
\text { como aproximadamente } 75 \% \text { de las muertes } \\
\text { neonatales, se pueden evitar con atención } \\
\text { oportuna. }\end{array}$ \\
\hline $\begin{array}{l}\text { Dansereau y } \\
\text { col., } 2018\end{array}$ & $\begin{array}{l}\text { Mesoamér } \\
\text { ica }\end{array}$ & $\begin{array}{l}\text { Cobertura } \quad \text { y } \\
\text { oportunidad de la } \\
\text { atención prenatal en } \\
\text { mujeres pobres de } 6 \\
\text { países de Mesoamérica }\end{array}$ & $\begin{array}{l}\text { Aproximadamente el } 9 \% \text { de las mujeres } \\
\text { gestantes con baja asistencia prenatal } \\
\text { (condiciones de pobreza, bajo nivel de } \\
\text { instrucción, atención sanitaria no calificada) } \\
\text { reportó haber sufrido complicaciones como: } \\
\text { nacimiento de feto muerto o aborto natural y un } \\
13 \% \text { hemorragia. }\end{array}$ \\
\hline
\end{tabular}




\begin{tabular}{|c|c|c|c|}
\hline $\begin{array}{ll}\text { Andrade } & \text { y } \\
\text { col., } 2019 & \end{array}$ & Brasil & $\begin{array}{l}\text { Atención prenatal en } \\
\text { grupo: efectividad y } \\
\text { retos de } \quad \text { su } \\
\text { implementación }\end{array}$ & $\begin{array}{l}\text { La atención prenatal adecuada y calificada } \\
\text { mejora los resultados materno-infantiles y reduce } \\
\text { significativamente las complicaciones durante el } \\
\text { embarazo, parto y puerperio sobre todo la } \\
\text { mortalidad materna. }\end{array}$ \\
\hline $\begin{array}{l}\text { Hernández y } \\
\text { col., } 2019\end{array}$ & Perú & $\begin{array}{l}\text { Factores asociados a la } \\
\text { calidad de la atención } \\
\text { prenatal en Perú }\end{array}$ & $\begin{array}{l}\text { En Perú las complicaciones obstétricas (la } \\
\text { mortalidad materna es la más importante) han } \\
\text { experimentado un decrecimiento marcado en las } \\
\text { últimas décadas debido a que el } 88,9 \% \text { de } \\
\text { mujeres recibió al menos } 6 \text { controles prenatales. } \\
\text { Las complicaciones obstétricas se presentan en } \\
\text { habitantes de zona rural, sierra y selva, zonas de } \\
\text { pobreza por acceso limitado a asistencia } \\
\text { prenatal. }\end{array}$ \\
\hline $\begin{array}{l}\text { Juárez y col., } \\
2020\end{array}$ & México & $\begin{array}{l}\text { Barreras en mujeres } \\
\text { indígenas para acceder } \\
\text { a servicios obstétricos } \\
\text { en el marco de redes } \\
\text { integradas de servicio } \\
\text { de salud }\end{array}$ & $\begin{array}{l}\text { Casi el } 20 \% \text { de las mujeres que no iniciaron } \\
\text { control prenatal en el primer trimestre del } \\
\text { embarazo presentaron complicaciones durante el } \\
\text { periodo gestacional y de parto entre ellas la } \\
\text { pérdida del latido fetal }(7,7 \%) \text {, falta de dilatación } \\
(7,7 \%) \text {, rotura prematura de membranas }(7,7 \%) \text {, } \\
\text { parto fortuito }(7,7 \%) \text {, alumbramiento tardío } \\
(7,7 \%) \text {, preeclampsia }(26,9) \text {, posición anómala } \\
(7,7 \%) \text { y hemorragia }(11,5 \%) \text {. }\end{array}$ \\
\hline $\begin{array}{l}\text { Betancourt y } \\
\text { col., } 2020\end{array}$ & Venezuela & $\begin{array}{l}\text { Factores asociados a la } \\
\text { no adherencia del } \\
\text { control prenatal en } \\
\text { gestantes }\end{array}$ & $\begin{array}{l}\text { La atención prenatal reduce la morbilidad y } \\
\text { mortalidad materna y perinatal, partos } \\
\text { prematuros, numero de productos con bajo peso } \\
\text { al nacer, además permite identificar factores de } \\
\text { riesgo para intervenciones oportunas. La falta de } \\
\text { control prenatal se debe al factor económico, } \\
\text { transporte, distancia de unidades de salud, } \\
\text { asignación tardía de citas y deficiente } \\
\text { conocimiento. }\end{array}$ \\
\hline Piña, 2020 & Ecuador & $\begin{array}{l}\text { Determinantes sociales } \\
\text { de la salud que influyen } \\
\text { en la adherencia al } \\
\text { control prenatal }\end{array}$ & $\begin{array}{l}\text { La falta de adherencia al control prenatal por } \\
\text { factores como la edad temprana, creencias, } \\
\text { costumbres, falta de accesibilidad geográfica, } \\
\text { clima de la región y desigualdad de género } \\
\text { suscita el aparecimiento de complicaciones que } \\
\text { pueden dejar secuelas en la madre y en la } \\
\text { comunidad en general. }\end{array}$ \\
\hline $\begin{array}{ll}\text { Miranda } & \text { y } \\
\text { col., } 2016\end{array}$ & Colombia & $\begin{array}{l}\text { Factores de necesidad } \\
\text { asociados al uso } \\
\text { adecuado del control } \\
\text { prenatal }\end{array}$ & $\begin{array}{l}\text { La mayoría de gestantes son jóvenes y pese a } \\
\text { estar en condiciones de vulnerabilidad } \\
\text { económica y educativa hacen uso adecuado del } \\
\text { control prenatal lo que ha repercutido en la poca } \\
\text { incidencia de complicaciones gestacionales y el } \\
\text { éxito del embarazo }\end{array}$ \\
\hline $\begin{array}{l}\text { Ministerio de } \\
\text { Salud } \\
\text { Pública, } 2015\end{array}$ & Ecuador & Guía de Práctica clínica & $\begin{array}{l}\text { Las principales causas de mortalidad materna y } \\
\text { perinatal son detectables y prevenibles con un } \\
\text { control prenatal temprano, oportuno y de alta } \\
\text { calidad porque permite identificar y controlas los } \\
\text { factores de riesgo obstétricos. El control prenatal } \\
\text { no solo reduce la mortalidad y discapacidad } \\
\text { infantil sino también contribuye a mejorar la salud } \\
\text { y calidad de vida de mujeres, familia y } \\
\text { comunidades. }\end{array}$ \\
\hline $\begin{array}{l}\text { Martínez J \& } \\
\text { Pererira M., } \\
2015\end{array}$ & Colombia & $\begin{array}{l}\text { Caracterización de las } \\
\text { gestantes de alto riesgo } \\
\text { obstétrico en el } \\
\text { departamento de Sucre }\end{array}$ & $\begin{array}{l}\text { La mayoría de gestantes en condiciones } \\
\text { psicosociales desfavorables llegan a programas } \\
\text { de atención prenatal en estados de gestación } \\
\text { avanzados ( } 30-40 \text { semanas) lo que se relaciona } \\
\text { con un mayor número de complicaciones como } \\
\text { parto pretérmino }(13,8 \%) \text {, aborto }(22,8 \%) \text {. }\end{array}$ \\
\hline $\begin{array}{l}\text { Corona B. y } \\
\text { col.,2015 }\end{array}$ & Cuba & $\begin{array}{l}\text { Caracterización de los } \\
\text { procesos de atención } \\
\text { prenatal a las gestantes }\end{array}$ & $\begin{array}{l}\text { Las infecciones vaginales durante el embarazo } \\
\text { constituyen un grave problema de salud por las } \\
\text { complicaciones obstétricas que producen como: }\end{array}$ \\
\hline
\end{tabular}




\begin{tabular}{|c|c|c|c|}
\hline & & $\begin{array}{l}\text { con morbilidad materna } \\
\text { extremadamente grave }\end{array}$ & $\begin{array}{l}\text { rotura prematura de membrana, parto pretérmino } \\
\text { y bajo peso debidas a una débil ejecución de } \\
\text { acciones de prevención en la atención prenatal }\end{array}$ \\
\hline $\begin{array}{l}\text { Melo y col., } \\
2015\end{array}$ & Brasil & $\begin{array}{l}\text { Factors associated with } \\
\text { the quality of prenatal } \\
\text { care: an approach to } \\
\text { premature birth }\end{array}$ & $\begin{array}{l}\text { Los nacimientos prematuros y la mortalidad } \\
\text { materno-infantil pueden prevenirse con una } \\
\text { atención prenatal de calidad y una observación } \\
\text { rigurosa de los criterios recomendados por el } \\
\text { Ministerio de Salud de Brasil y los programas } \\
\text { nacionales y estatales. }\end{array}$ \\
\hline $\begin{array}{ll}\text { Morales } & y \\
\text { col., } 2018\end{array}$ & México & $\begin{array}{l}\text { Epidemiología de la } \\
\text { muerte materna e } \\
\text { México y el } \\
\text { cumplimiento } \quad \text { del } \\
\text { objetivo } 5 \text { del Desarrollo } \\
\text { del milenio, hacia los } \\
\text { objetivos de desarrollo } \\
\text { sostenible }\end{array}$ & $\begin{array}{l}303 \text { mil mujeres han muerto por complicaciones } \\
\text { obstétricas (hemorragia grave, infecciones en } \\
\text { puerperio, hipertensión) durante el embarazo, } \\
\text { parto y puerperio, mismas que en su mayoría } \\
\text { eran prevenibles con un control prenatal } \\
\text { adecuado. }\end{array}$ \\
\hline $\begin{array}{l}\text { Nava y col., } \\
2020\end{array}$ & México & $\begin{array}{l}\text { Morbilidad materna } \\
\text { extrema: intervenciones } \\
\text { médico-quirúrgicas e } \\
\text { indicadores para evitar } \\
\text { la muerte materna }\end{array}$ & $\begin{array}{l}\text { Una de las estrategias más efectivas para } \\
\text { prevenir complicaciones como las hemorragias y } \\
\text { trastornos hipertensivos es el control prenatal } \\
\text { activo sobretodo del tercer periodo de gestación. }\end{array}$ \\
\hline $\begin{array}{l}\text { Salazar } \quad \text { E. } \\
2019\end{array}$ & Perú & $\begin{array}{ll}\text { El problema de la } \\
\text { seguridad de la } \\
\text { atención obstétrica }\end{array}$ & $\begin{array}{l}\text { Las deficiencias en el control prenatal (calidad de } \\
\text { atención, inasistencia, asistencia tardía a } \\
\text { consulta) llevan a complicaciones en el } \\
\text { embarazo, como la infección urinaria }(2-10 \%) \text { y } \\
25 \text { a } 30 \% \text { de estas mujeres desarrollan } \\
\text { pielonefritis, como consecuencia existe un } \\
\text { incremento de patologías agregadas como el } \\
\text { bajo peso al nacer y la prematuridad. }\end{array}$ \\
\hline $\begin{array}{l}\text { Naula y col., } \\
2019\end{array}$ & Ecuador & $\begin{array}{l}\text { Complicaciones del } \\
\text { puerperio inmediato en } \\
\text { pacientes atendidas en } \\
\text { el Servicio de } \\
\text { Ginecología } \quad \text { y } \\
\text { obstetricia del Hospital } \\
\text { Vicente Corral Moscoso }\end{array}$ & $\begin{array}{l}\text { El } 84,4 \% \text { de estantes asistió a consulta prenatal } \\
\text { debido a la ampliación de la red de atención } \\
\text { prenatal nacional lo que redujo la incidencia de } \\
\text { complicaciones obstétricas como la hipotonía, } \\
\text { anemia, atonía uterina, desgarros y } \\
\text { alumbramiento incompleto, además de la } \\
\text { mortalidad materna a un } 0,5 \%\end{array}$ \\
\hline $\begin{array}{l}\text { Ipia y col., } \\
2019\end{array}$ & Colombia & $\begin{array}{l}\text { Impacto de las } \\
\text { condiciones } \\
\text { sociodemográficas } \\
\text { sobre el control prenatal } \\
\text { en Latinoamérica }\end{array}$ & $\begin{array}{l}\text { La mayoría de las defunciones maternas se } \\
\text { origina por las complicaciones obstétricas, las } \\
\text { cuales son evitables por medio de intervenciones } \\
\text { médicas oportunas, es decir son susceptibles de } \\
\text { ser intervenidas durante el control prenatal. }\end{array}$ \\
\hline $\begin{array}{l}\text { Izaguirre y } \\
\text { col., } 2016\end{array}$ & Honduras & $\begin{array}{l}\text { Incremento del riesgo } \\
\text { obstétrico en embarazo } \\
\text { adolescente. Estudio de } \\
\text { casos y controles }\end{array}$ & $\begin{array}{l}\text { Las gestantes que provienen de áreas rurales o } \\
\text { con limitado acceso a atención de salud prenatal } \\
\text { tienen dos veces más riesgo de presentar } \\
\text { complicaciones obstétricas en relación a las del } \\
\text { área urbana durante el embarazo, preparto, } \\
\text { parto, puerperio y periodo neonatal. Las } \\
\text { principales complicaciones obstétricas } \\
\text { reportadas fueron: infecciones urinarias, estado } \\
\text { fetal no tranquilizador, ruptura prematura de } \\
\text { membranas, vaginitis/vaginosis, anemia, } \\
\text { desgarros, histerectomía y hemorragia. }\end{array}$ \\
\hline
\end{tabular}

\section{DISCUSIÓN}

El control prenatal es un servicio de salud que tiene el propósito de detectar las alteraciones del embarazo para impactar de manera positiva en la reducción de la mortalidad materna e infantil, en especial en países de alta vulnerabilidad como los latinoamericanos donde las mujeres no asisten a controles prenatales debido a varias circunstancias como el bajo nivel de instrucción, ocupaciones del hogar y crianza, falta de empleo, escasos recursos económicos, ubicación geográfica del hogar y el centro asistencial, falta de afiliación de seguridad social, además de factores actitudinales como creencias y costumbres o la baja calidad sanitaria $(5,10)$

La persistencia de estos factores limita cada vez más que las embarazadas acudan a recibir atención prenatal y consecuentemente presenten complicaciones obstétricas, argumento que se ha documentado en varias investigaciones latinoamericanas, varias de ellas realizadas en Colombia donde se asume que un buen control prenatal tiene un impacto positivo sobre el desenlace del embarazo pues la mayoría de 
complicaciones como el parto pretérmino (13,8\%), aborto $(22,8 \%)$, muertes maternas generalmente por infecciones y hemorragias, así como aproximadamente $75 \%$ de las muertes neonatales, se pueden evitar con atención gestacional oportuna $(11,12)$. Ipia y col., (13) afirman que la mayoría de complicaciones obstétricas y muerte materna son evitables por medio de intervenciones médicas oportunas, es decir son susceptibles de ser intervenidas durante el control prenatal. García y col., (14) añaden que las mujeres colombianas con escaso o nulo control prenatal o revisiones en etapas avanzadas de embarazo presentan mayor probabilidad de presentar complicaciones obstétricas que llevan al intervencionismo de emergencia (25\%) o a la pérdida fetal $(15 \%)$. En base a esta problemática organismos de salud trabajan por ampliar la cobertura de atención prenatal sobre todo en zonas de vulnerabilidad económica y educativa para lograr éxito en el embarazo (15).

Estudios realizados en Brasil, también demuestran la relación entre las complicaciones obstétricas y la falta de control prenatal, Melo y col. (16) realizaron una investigación en torno al cuidado prenatal en mujeres en el puerperio destacan que la adecuada atención prenatal es un factor determinante en la prevención de la morbimortalidad materna e infantil, ya que contribuye a obtener resultados más favorables de la realización de los procedimientos básicos como la realización de pruebas clínicas y de laboratorio y el seguimiento del embarazo mediante visitas periódicas para la detección y tratamiento oportuno de los factores de riesgo que traen complicaciones para la salud materno infantil. Estos autores además exponen que las asistencias regulares a controles prenatales pueden ser determinantes para el aparecimiento de problemas maternos y neonatales, entre ellos las malformaciones congénitas, infecciones, preeclampsia y eclampsia, escasa ganancia de peso fetal y parto prematuro considerada internacionalmente uno de los más importantes problemas de salud pública actual. Andrade (17) apoya firmemente esta evidencia y alude que la atención prenatal adecuada y calificada mejora indiscutiblemente los resultados materno-infantiles al reducir significativamente las complicaciones durante el embarazo, parto y puerperio sobre todo la mortalidad materna.

En México, Juárez y col. (18) encontraron resultados similares a los reportados en Colombia y Brasil respecto al riesgo de complicaciones obstétricas por falta de atención de salud gestacional al encontrar que casi el $20 \%$ de las mujeres que no iniciaron control prenatal en el primer trimestre del embarazo presentaron complicaciones durante el periodo gestacional y de parto entre ellas la pérdida del latido fetal $(7,7 \%)$, falta de dilatación $(7,7 \%)$, rotura prematura de membranas $(7,7 \%)$, parto fortuito $(7,7 \%)$, alumbramiento tardío $(7,7 \%)$, preeclampsia $(26,9)$, posición anómala $(7,7 \%)$ y hemorragia (11,5\%). De ahí que autores como Nava y col., (19) sostienen que una de las estrategias más efectivas para prevenir complicaciones obstétricas es el control prenatal activo sobretodo en el primer y tercer periodo de gestación. Solo con atención prenatal oportuna, regular y de calidad se puede evitar que miles de vidas se pierdan cada año por causas prevenibles (infecciones, hemorragias) sobre todo en zonas menos favorecidas donde aún persiste inequidad en el acceso a servicios de salud (20).

En Cuba, el control prenatal es reconocido como uno de los instrumentos de prevención de morbilidad y mortalidad materno-infantil más importante, porque a través de él se pueden identificar los factores de riesgo en la gestante y enfermedades que pueden afectar el trascurso normal del embarazo o a su vez comprometer la salud del recién nacido entre las más comunes están la infección vaginal, la preeclampsia, hemorragia posparto, trastornos hipertensivos, enfermedad tromboembolia y la sepsis, problemas obstétricos que pueden desencadenar lesiones permanentes que muchas veces se traducen en discapacidades o muerte (21).

Un estudio hondureño en el que se determinó los riesgos obstétricos en una muestra de 1354 adolescentes embarazadas se encontró que las gestantes que provienen de áreas rurales tienen dos veces más riesgo de presentar complicaciones durante el embarazo, entre ellas las infecciones urinarias, estado fetal no tranquilizador, ruptura prematura de membranas, vaginitis/vaginosis, anemia y durante el parto y posparto de presentar desgarros, histerectomía y hemorragia debido a la escasa atención prenatal recibida (22). Lo expuesto coincide con un estudio Venezolano, desarrollado por Betancourt y col., (23) en el que se menciona que la atención prenatal reduce significativamente la morbilidad y mortalidad materna y neonatal, partos prematuros y el número de productos con bajo peso al nacer, ello debido a la facilidad de esta estrategia de salud pública para identificar los factores de riesgo que hace posible plantear acciones preventivas y terapéuticas oportunas durante el embarazo, lo que se traduce en menor costo en su atención posterior y en una mejora de la calidad de vida de las gestantes y sus hijos a largo plazo.

En Perú, también se han llevado a cabo investigaciones en torno a la importancia del control prenatal para prevenir las complicaciones obstétricas y la muerte materno-infantil, una de ellas es la de Núñez y col., (24) en la que se encontró que el $39,39 \%$ de las gestantes con un control prenatal incompleto presentó una inadecuada ganancia de peso, infecciones urinarias y la anemia con lo que asumen que el número de complicaciones obstétricas es mayor en gestantes con un control prenatal inadecuado o nulo. Resultado que es apoyado por Cardozo y col., (25) quien tras analizar las causas de las complicaciones y morbilidad obstétrica en gestantes encontró que esto se debe al incumplimiento de controles mínimos recomendados pues tanto las hemorragias, infecciones y cuadros hipertensivos son prevenibles con chequeos continuos y tratamientos específicos. A más de ello Salazar (26) añade que las deficiencias en el control prenatal debidas a la calidad de atención, inasistencia o asistencia tardía a consulta llevan al aparecimiento de complicaciones en el embarazo, como la infección urinaria (2-10\%) y del 25 a $30 \%$ al desarrollo de pielonefritis dando como resultado un incremento de patologías obstétricas agregadas como el bajo peso al nacer y la prematuridad.

Ecuador al igual que otras zonas latinoamericanas ha puesto especial interés en la prevención de las complicaciones obstétricas en base al control prenatal y perinatal; muestra de aquello es la investigación de Piña (27) en la que se manifiesta que falta de adherencia al control prenatal por factores como la edad temprana, creencias, costumbres, falta de accesibilidad geográfica, clima de la región y desigualdad de género suscita el aparecimiento de complicaciones que pueden dejar secuelas permanentes en la madre, el neonato y afectar al contexto familiar y social. Estos hallazgos, también se sustentan en los de Naula y col., quienes en 2019 desarrollaron una investigación basada en historias clínica y encuestas sociodemográficas en una población de 365 pacientes, encontrándose que, el $84,4 \%$ asistió a consulta prenatal debido a la ampliación de la red de atención prenatal nacional lo que redujo la incidencia de complicaciones obstétricas como la hipotonía, anemia, atonía uterina, desgarros y alumbramiento incompleto, además de la mortalidad materna a un $0,5 \%$ (28). Es por ello que el Ministerio 
de Salud Pública teniendo como base que las principales causas de mortalidad materna y perinatal en el país son detectables y prevenibles con un control prenatal temprano, oportuno y de alta calidad maneja campañas y programas de seguimiento gestacional intensivas ya que el control prenata no solo reduce la mortalidad y discapacidad infantil sino también contribuye a mejorar la salud y calidad de vida de mujeres, familia y comunidades (29).

Por estas razones, es posible afirmar la relación que tiene la falta de control prenatal y el aparecimiento de las complicaciones obstétricas pues como lo ostentan Hernández y col., (30) las complicaciones obstétricas (la mortalidad materna es la más importante) han experimentado un decrecimiento marcado en las últimas décadas debido a que el $88,9 \%$ de mujeres recibe al menos 6 controles prenatales a cargo de profesionales calificados, lo que resulta alentador porque al lograr asistencia de salud en la gestación se pueden garantizar partos más satisfactorios.

\section{CONCLUSIONES}

El embarazo es un evento fisiológico normal, pero debido a las complicaciones obstétricas que pueden derivarse de este periodo y afectar a la madre y al neonato por las difíciles condiciones socioeconómicas de las gestantes latinoamericanas, se considera que la gestación debe ser vigilada mediante un control prenatal adecuado, que en la mayoría de los caso no se da debido al bajo nivel de instrucción, ocupaciones del hogar y crianza, falta de empleo, escasos recursos económicos, ubicación geográfica del hogar y el centro asistencial, falta de afiliación de seguridad social, además de factores actitudinales como creencias y costumbres específicas en grupos socioculturales, pero que de no abordarse puede traer graves consecuencias tanto para la madre como para el niño, la familia y la comunidad en general, ya que como se ha demostrado con la revisión de varios estudios, existe una relación directa entre la falta de control prenatal y el aparecimiento y complejidad de complicaciones obstétricas.

De acuerdo a investigaciones realizadas en países como Colombia Brasil, México, Cuba, Venezuela, Perú y Ecuador en los últimos años, se ha comprobado que el control prenatal deficiente, incompleto o nulo, es un indicador significativo de malos resultados obstétricos tanto a corto como a largo plazo porque el hecho de carecer de procedimientos diagnósticos que ayuden a vigilar la salud materno-fetal produce una falsa sensación de seguridad o de bajo riesgo que puede complicar el embarazo y el parto, donde se evidencian complicaciones como: las infecciones del tracto urinario, anemia, alteraciones de la presión, hemorragias, desgarros perineales, alumbramiento incompleto y en el neonato, el bajo peso y la prematuridad, y en ambos casos la probabilidad de discapacidad y muerte debido a la escasa monitorización, así como la ejecución de procedimientos terapéuticos inoportunos que dificultan que el embarazo llegue a feliz término.

\section{CONFLICTO DE INTERESES}

Ninguno declarado por los autores

\section{FINANCIAMIENTO}

Autofinanciamiento

\section{AGRADECIMIENTOS}

Ninguno

\section{REFERENCIAS}

1. Organización Mundial de la salud. Mortalidad materna. Informativo. Centro de Prensa; 2019. Disponible en: https://www.who.int/es/news-room/fact-sheets/detail/maternal-mortality

2. Naciones Unidas. Panorama de la Salud: Latinoamérica y el Caribe 2020. Disponible en: https://www.oecdilibrary.org/sites/bcb4ab39-es/index.html?itemld=/content/component/bcb4ab39-es

3. UNFPA Latinaoamerica. Overview of the situation of maternal morbidity and mortality: Latin america and caribbean; 2017. Disponible en: https://lac.unfpa.org/sites/default/files/pub-pdf/MSH-GTR-Report-Eng.pdf

4. Rojas J, Suárez N, Ateka O. Developing obstetric medicine training in Latin America. Obstetric Medicine. 2017; 10(1): 16-20. Disponible https://journals.sagepub.com/doi/pdf/10.1177/1753495X16684708\#articleCitationDownloadContainer

5. Castro A. Witnessing Obstetric Violence during Fieldwork. Health and Human Rights Journal. 2019; $21(1): 103-11$. Disponible en: https://www.ncbi.nlm.nih.gov/pmc/articles/PMC6586976/

6. Cáceres M, Ruíz M. Prevalencia de inicio de la atención prenatal. Asociación con el nivel socioeconómico de la gestante. Revista Colombiana de Obstetricia y Ginecología. 2018; 69(1):22-31. Disponible en: http://www.scielo.org.co/pdf/rcog/v69n1/es_2463-0225-rcog-69-01-00022.pdf

7. Fernandez-Chinguel J, Zafra-Tanaka J, Goicochea-Lugo S, Peralta C, Taype-Rondan A. Aspectos básicos sobre la lectura de revisiones sistemáticas y la interpretación de meta-análisis. Acta Med Peru. 2019; 36(2):157-69. Disponible en: http://www.scielo.org.pe/pdf/amp/v36n2/a13v36n2.pdf

8. Carrion J, Correa A, Alvarado F. El MeSH y la pregunta pico. Una herramienta clave para la búsqueda de información. Revista Científico-sanitaria. https://www.revistacientificasanum.com/pdf/sanum_v4_n1_a5.pdf

9. Moher D, Shamseer L, Clarke M, Ghersi D, Liberati A, Petticrew M. Ítems de referencia para publicar Protocolos de Revisiones Sistemáticas y Metaanálisis: Declaración PRISMA-P 2015. Revista Española de Nutrición Humana y Dietética. 2016; 20(2):148-160. Disponible en: http://renhyd.org/index.php/renhyd/article/view/223

10. Dansereau E, McNellan C, Gagnier M, Sima S, Haakenstad A, Casey J. Cobertura y oportunidad de la atención prenatal en mujeres pobres de 6 países de Mesoamérica. Banco Interamericano de Desarrollo. 2018: 3-15. Disponible en: https://publications.iadb.org/publications/spanish/document/Cobertura-y-oportunidad-de-la-atenci\%C3\%B3n-prenatalen-mujeres-pobres-de-6-pa\%C3\%ADses-de-Mesoam\%C3\%A9rica.pdf 
11. Martínez J, Pereira M. Caracterización de las gestantes de alto riesgo obstétrico ARO en el departamento de Sucre Colombia. Salud Uninorte. 2015; 32(3):452-460. Disponible en: https://www.redalyc.org/pdf/817/81750089008.pdf

12. Gómez F, Marrugo C, Florez A, López K, Mazzanett E, Orozco J. Impacto económico y sanitario del control prenatal en embarazadas afiliadas al régimen subsidiado en Colombia durante 2014. Revista de salud pública de México. 2017; 59(2):176-180. Disponible en:

13. Ipia N, Ortega D, Acosta P, López W, Martínez J, Corrales N. Impacto de las condiciones sociodemográficas sobre el control prenatal en Latinoamérica. Revista de la Facultad de Medicina. 2019; 67(3): 331-335. Disponible en: https://media.proquest.com/media/hms/PFT/1/46ZtB?_s=meE0QjDs7SIDCmw6qhSds8vfHDw\%3D

14. García C. Barreras de acceso y calidad en el control prenatal. Revista de la Facultad de Medicina. 2017; 65(2):3015310. Disponible en: http://www.scielo.org.co/pdf/rfmun/v65n2/0120-0011-rfmun-65-02-305.pdf

15. Miranda C, Castillo I. Factores de necesidad asociados al uso adecuado del control prenatal. Revista Cuidarte. 2016; 7(2):1345-1351. Disponible en: https://www.redalyc.org/pdf/3595/359546229012.pdf

16. Melo E, Rosseto R, Freitas T. Factors associated with the quality of prenatal care: an approach to premature birth. Revista da Escola de Enfermagem da USP. 2015; 49(4):540-548. Disponible en: https://www.scielo.br/pdf/reeusp/v49n4/0080-6234-reeusp-49-04-0540.pdf

17. Andrade Z, Heredia I, Fuentes E, Alcalde J, Bravo L, Jurkiewicz L. Atención prenatal en grupo: efectividad y retos de su implementación. Revista de Saúde Pública. 2019 Septiembre; 53(85):1-8. Disponible en: https://www.scielo.br/scielo.php?pid=S0034-89102019000100604\&script=sci_arttext\&tlng=es

18. Juárez C, Villalobos A, Sauceda A, Nigenda G. Barreras en mujeres indígenas para acceder a servicios obstétricos en el marco de redes integradas de servicios de salud. Gaceta Sanitaria. 2020; 34(6):546-552. Disponible en: https://www.sciencedirect.com/science/article/pii/S0213911119301621

19. Nava E, González L, Salcedo A, Cisneros F, Dávila J, Durán A. Morbilidad materna extrema: intervenciones médicoquirúrgicas e indicadores para evitar la muerte materna. Ginecol Obstet Mex. 2020; 88(9):606-614. Disponible en: https://www.medigraphic.com/pdfs/ginobsmex/gom-2020/gom209f.pdf

20. Morales E, Ayala I, Morales H, Astorga M, Castro A. Epidemiologia de la muerte materna en México y el cumplimiento del Objetivo 5 de desarrollo del milenio, hacia los objetivos de desarrollo sostenible. Revista de Especialidades Médico Quirúrgicas. 2018; 23:61-86. Disponible en: https://www.medigraphic.com/pdfs/quirurgicas/rmq-2018/rmq182a.pdf

21. Corona B, Pérez D, Espinoza R, Álvarez M, Salvador S, Fernández L. Caracterización de los procesos de atención prenatal a las gestantes con morbilidad materna extremadamente grave. Revista Cubana de Higiene y Epidemiología. 2015; 53(1):303. Disponible en: http://scielo.sld.cu/scielo.php?script=sci_arttext\&pid=S1561-30032015000100002

22. Izaguirre A, Aguilar V, Ramírez A, Valladares G, Rivera W, Valladares E. Incremento del Riesgo Obstétrico en Embarazo Adolescente. Estudio de Casos y controles. ImedPub Journals. 2016; 12(3):1-6. Disponible en: https://www.archivosdemedicina.com/medicina-de-familia/incremento-del-riesgo-obsteacutetrico-en-embarazoadolescente-estudio-de-casos-y-controles.pdf

23. Betancourt A, García M. Factores asociados a la no adherencia del control prenatal en gestantes. Revista Arbitrada Interdisciplinaria de Ciencias de la Salud. 2020; 4(7):74-94. Disponible en: https://fundacionkoinonia.com.ve/ojs/index.php/saludyvida/article/view/646

24. Núñez R, Moquillaza V, García J. Complicaciones obstétricas asociadas al control prenatal inadecuado en puérperas atendidas en un centro materno infantil peruano. Perinatología y reproducción humana. 2019;(33):58-64. Disponible en: https://www.researchgate.net/publication/337966284_Complicaciones_obstetricas_asociadas_al_control_prenatal_ina decuado_en_puerperas_atendidas_en_un_centro_materno_infantil_peruano_2018

25. Cardozo D, Joaleef $Y$. Complicaciones obstétricas y morbilidad obstétrica en gestantes en el servicio Gíneco-obstetricia de un Hospital en la Ciudad de Lambayeque. Revista Científica Salud \& Vida Sipanense. 2015; 2(1):26-36. Disponible en: http://revistas.uss.edu.pe/index.php/SVS/article/view/80

26. Salazar E. El problema de la seguridad de la atención obstétrica. Revista Peruana de Ginecología y Obstetricia. 2019; 65(1):31-34. Disponible en: http://www.scielo.org.pe/pdf/rgo/v65n1/a06v65n1.pdf

27. Piña E. Determinantes sociales de la salud que influyen en la adherencia al control prenatal. Revista Publicando. 2020; 7(26):54-60. Disponible en: https://revistapublicando.org/revista/index.php/crv/article/view/2136/2207

28. Naula L, Quiñonez C, Solano B, Méndez J, Oviedo Y, Carpio D. Complicaciones del puerperio inmediato en pacientes atendidas en el servicio de ginecología y obstetricia del Hospital Vicente Corral Moscoso. Revista Latinoamericana de Hipertensión. 2019; 14(2):1-8. Disponible en: http://www.revhipertension.com/rlh_2_2019/complicaciones_puerperio_inmediato.pdf

29. Ministerio de Salud Pública. Control prenatal: Guía de Práctica Clínica. Primera ed. Quito: Dirección Nacional de Normatización; 2015. Disponible en: https://www.salud.gob.ec/wp-content/uploads/2014/05/GPC-CPN-final-mayo2016-DNN.pdf

30. Hernández A, Vargas R, Bendezu G. Factores asociados a la calidad de la atención prenatal en Perú. Revista Peruana de medicina experimental y salud pública. 2019; 36(2):178-187. Disponible en: https://scielosp.org/article/rpmesp/2019.v36n2/178-187/ 
